\title{
Two siblings with cerebrotendinous xanthomatosis
}

\section{C Kottahachchi ${ }^{1}$, B L P P Balasooriya ${ }^{1}$, L Panangala ${ }^{2}$, U K Ranawaka ${ }^{1}$}

Ceylon Medical Journal 2012; 57: 128-129

\section{Introduction}

Cerebrotendinous xanthomatosis (CTX) is a rare disorder of lipid metabolism. We report two siblings with this genetic disorder.

\section{Case report}

A 26-year old woman was referred for evaluation of progressive difficulty in walking which started at the age of 12 years. She had developed bilateral cataracts at the age of 15 . Examination showed bilateral pyramidal signs with cerebellar ataxia. She had xanthomas involving elbows, wrists, knees and ankles bilaterally. Further enquiry revealed a history of similar illness in a brother aged 31 . He had developed seizures at the age of 4 years, difficulty in walking at 5 years, and bilateral cataracts at 10 years. Examination showed cerebellar ataxia with pyramidal signs, facial dysmorphism due to facial xanthomas and peripheral xanthomas (Figure 1).

Investigations of both siblings showed normal serum cholesterol, fasting blood sugar, renal function, urinalysis and thyroid profiles. X-rays of chest, and bilateral knee and ankle joints were normal. MRI scanning showed symmetrical and heterogenous signal changes in cerebellar dentate nuclei bilaterally (hypointense in $\mathrm{T} 1$, and hyperintense in T2 and FLAIR sequences), which are characteristic of CTX (Figure 2).
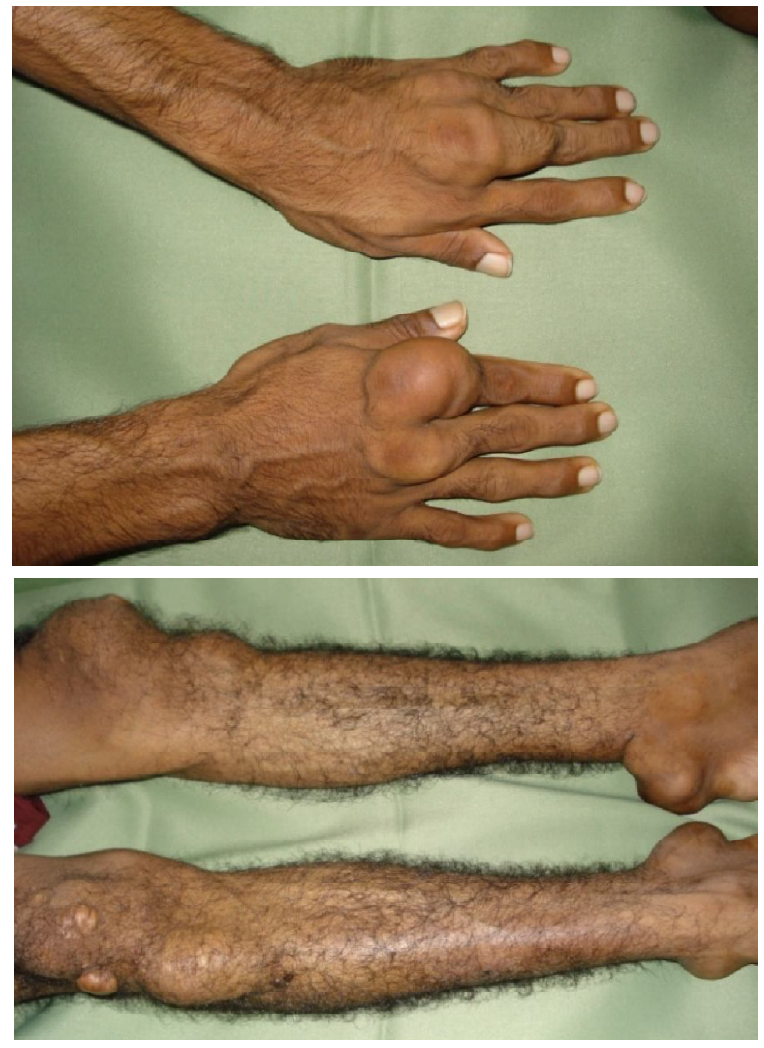

Figure 1. Tendon xanthomas in the brother.

${ }^{1}$ University Medical Unit, Teaching Hospital, Ragama and ${ }^{2}$ Rehabilitation Hospital, Ragama, Sri Lanka.

Correspondence: DCK, e-mail: <dula_k@yahoo.com>. Received 29 November 2011 and revised version accepted 13 January 2012. Competing interests: none declared. 

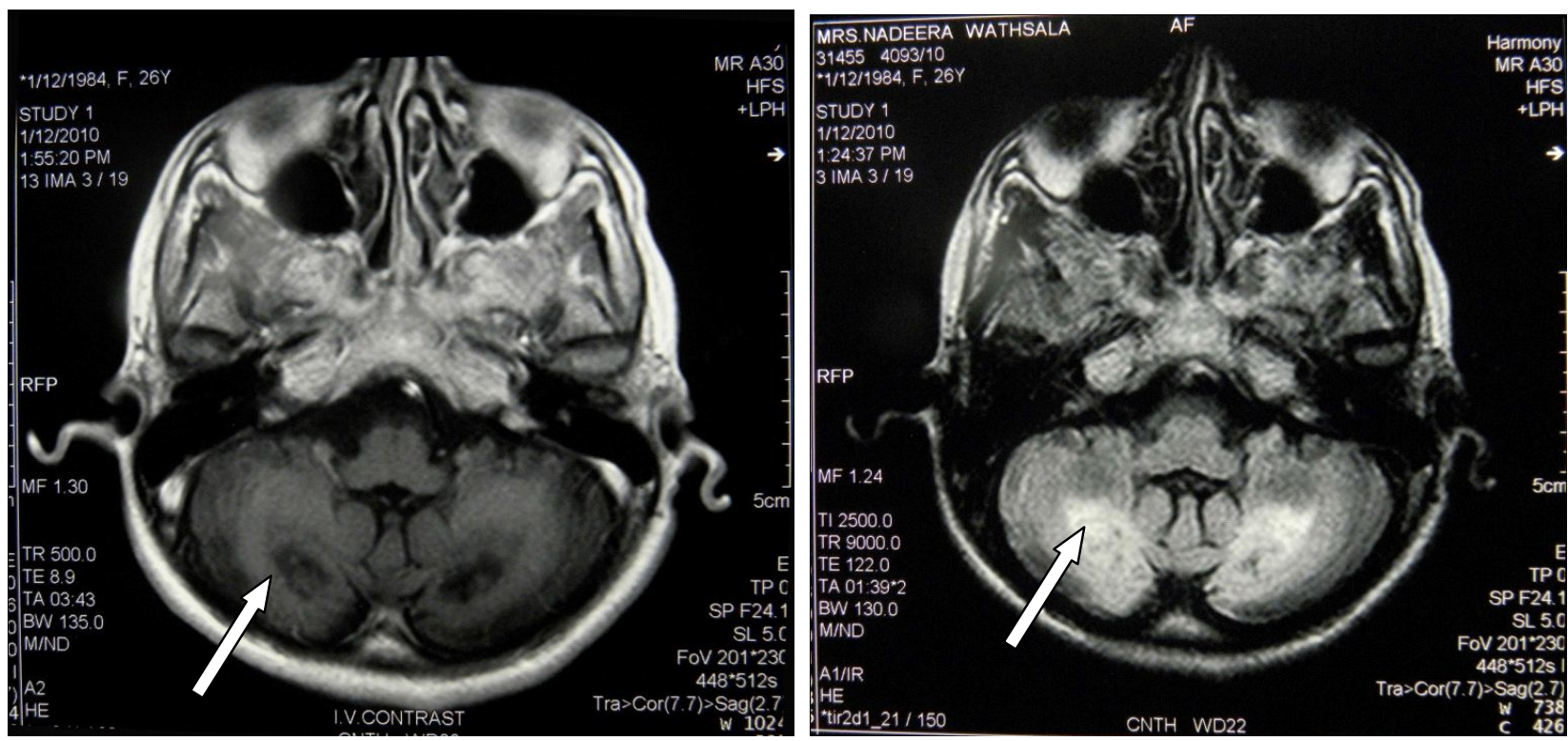

Figure 2. MRI Brain of sister showing characteristic appearance of dentate nuclei (hypointense in T1 and hyperintense in T2 weighted sequences).

\section{Discussion}

CTX is a rare autosomal recessive disorder of cholesterol and bile acid metabolism. Mutations in the sterol 27-hydroxylase gene result in diminished activity of this enzyme, a key enzyme in bile acid synthesis. This leads to reduced bile acid synthesis and marked accumulation of 7-alpha-hydroxylated bile acid precursors, especially 7-alpha-hydroxy-4-cholesten-3-one which is a precursor to cholestanol. Deposition of cholestanol and cholesterol in the brain and spinal cord, muscle (including the heart), lens, blood vessels and tendons results in progressive degenerative changes [1].

Patients develop presenile cataracts, other ocular features such as optic atrophy, and xanthomas that usually manifest after the age of 20. Children and young adults have low intelligence, but prominent neurologic symptoms develop in the third decade of life. These include mental retardation, dementia, spasticity, cerebellar ataxia, extrapyramidal features (dystonia, oromandibular dyskinesia, early onset Parkinson disease), seizures and peripheral neuropathy. Other manifestations include coronary and carotid vascular disease, bony changes (granulomatous bone lesions, osteopenia, osteoporosis, pathologic fractures) and endocrine disturbances (hypothyroidism).

Investigations include elevated cholestanol levels and low-to-normal serum cholesterol levels. A low cholesterol-to-cholestanol ratio is diagnostic in the appropriate clinical setting [2]. MRI findings of T2 hyperintensity of the dentate nuclei are characteristic. Other changes include diffuse atrophy and focal lesions in cerebellum, basal ganglia and cerebrum [3].

Early diagnosis of CTX is imperative because it is treatable. Chenodeoxycholic acid (CDCA) replacement is shown to improve bile acid metabolism, reduce serum cholestanol levels, and to retard disease progression [4]. Neuro-psychological and bone symptoms improve, but xanthoma and cataract development are not affected. If biochemical changes are not controlled with CDCA treatment alone, HMG-CoA reductase inhibitors may be added.

The siblings described had typical features of the syndrome with early learning difficulties, presenile cataracts, xanthomas, prominent neurological features and characteristic MRI findings. We were unable to perform serum cholestanol levels and genetic analysis (not available in Sri Lanka). They were treated with ursodeoxycholic acid as an alternative to CDCA (not available in Sri Lanka) and atorvastatin, and are on regular clinic follow up [5].

\section{References}

1. Cali JJ, Hsieh CL, Francke U, Russell DW. Mutations in the bile acid biosynthetic enzyme sterol 27-hydroxylase underlie cerebrotendinous xanthomatosis. Journal of Biological Chemistry 1991; 266: 7779.

2. Moghadasian MH. Cerebrotendinous xanthomatosis: clinical course, genotypes and metabolic backgrounds. Clinical and Investigative Medicine 2004; 27: 42.

3. Barkhof F, Verrips A, Wesseling P. Cerebrotendinous xanthomatosis: the spectrum of imaging findings and the correlation with neuropathologic findings. Radiology 2000; 217: 869 .

4. Samenuk P, Koffman BM. Chenodeoxycholic treatment of cerebrotendinous xanthomatosis. Neurology 2001; 56: 695.

5. Kimura S, Beppu T, Kugai N, et al. A case of cerebrotendinous xanthomatosis: effects of ursodeoxycholic acid administration on serum bile acids and cholestanol. Japanese Journal of Medicine 1982; 2: 210. 